\title{
Quais ações técnico-táticas realizadas durante as partidas de futsal podem discriminar o resultado de vitória ou derrota?
}

CDD. 20.ed. 796.073

796.331

http://dx.doi.org/10.1590/1807-55092014000200203

\author{
Bernardo MILOSKI* \\ João Pedro PINHO* \\ Camila Gobo de FREITAS* \\ Pablo Rebouças MARCELINO* \\ Ademir Felipe Schultz de ARRUDA*
}

\section{Resumo}

0 objetivo do presente estudo foi verificar se as ações técnico-táticas específicas do futsal são capazes de discriminar o resultado de partidas de uma equipe de alto rendimento. Foram analisadas 40 partidas de uma equipe de futsal durante dois anos de disputa da Liga Nacional. Considerou-se os resultados de vitória ou derrota. Durante cada partida, foram analisados o número total das seguintes ações técnicotáticas: Desarmes Realizados, Desarmes Sofridos, Total de Finalizações Realizadas, Total de Finalizações Sofridas, Finalizações Certas Realizadas, Finalizações Certas Sofridas, Passes Incompletos Realizados e Passes Incompletos do Adversário; bem como os índices de coeficiente para cada ação técnico-tática, a partir da razão entre ação realizada e sofrida. Foi realizada uma Análise Discriminante a partir do método de estimação simultânea. Apenas as variáveis Desarmes Realizados, Desarmes Sofridos e Coeficiente de Desarme apresentaram cargas discriminantes acima de 0,40 , indicando que as mesmas poderiam discriminar o resultado de vitória e derrota em partidas de futsal. Pode-se concluir que a eficiência em relação ao número de Desarmes Realizados e de Desarmes Sofridos pode ser considerada um fator importante para alcançar o resultado de vitória em partidas de futsal de alto rendimento. Este estudo apontou que o sistema defensivo da equipe de futsal analisada, tanto no que se refere à organização tática quanto no tocante à técnica adequada para realização do desarme se apresentou como um fator discriminante do resultado da partida. Além disso, o sistema ofensivo também parece ser importante no sentido de prover uma melhor organização de ataque enquanto diminui a possibilidade de sofrer desarmes.

Palavras-chave: Análise de desempenho; Esporte coletivo; Esporte de alto rendimento; Desempenho esportivo; Futsal.

\section{Introdução}

O futsal é uma versão "indoor" (ou de quadra) do futebol, reconhecido pela Fédération Internationale de Football Association (FIFA) ${ }^{1}$. Esta modalidade tem apresentado grande crescimento de popularidade, sendo jogada em ligas profissionais e amadoras, por homens e mulheres, ao redor do mundo ${ }^{2-3}$. Devido a esse grande aumento no número de participantes e países que adotaram a prática dessa modalidade, a partir do ano de 1989, a FIFA passou a organizar o Campeonato Mundial de Futsal, disputado por 16 seleçóes nacionais e realizado a cada quatro $\operatorname{anos}^{1-2}$. O Brasil é o país de maior destaque no cenário internacional, tendo conquistado cinco títulos mundiais reconhecidos pela FIFA.

O incremento da competitividade e o desenvolvimento da modalidade no cenário internacional têm atraído à atenção de pesquisadores da área do esporte. Assim, paralelamente ao crescimento da popularidade do futsal, nos últimos anos tem-se observado também um incremento no número de publicaçóes referentes à modalidade, notadamente no que diz respeito às demandas fisiológicas do esporte e as implicaçôes do nível de aptidão física dos atletas no desempenho competitivo ${ }^{1,3-5}$. 
Estes estudos têm revelado a importância de determinados atributos da aptidão física no nível de desempenho dos jogadores de futsal ${ }^{6-7}$. Apesar da relevância de se compreender o papel de atributos físicos no nível de desempenho competitivo no futsal, outros fatores devem ser considerados para o melhor entendimento da modalidade, considerando a natureza multifacetada do desempenho no esporte coletivo ${ }^{8-10}$. Dentre esses fatores, é imperativo considerar as ações técnicas / táticas específicas da modalidade, as quais se constituem em aspectos determinantes para o sucesso competitivo no esporte coletivo ${ }^{8-11}$.

Nesse sentido, a técnica pode ser entendida como a maneira de se executar uma tarefa, baseado em um padrão de movimento adequado, mas que respeite a individualidade do atleta ${ }^{12}$, buscando um resultado eficiente para a tarefa desempenhada. Por outro lado, os princípios táticos se referem a uma construção teórica relacionada à lógica do $\mathrm{jogo}^{13}$, consistindo de uma organização do plano de ações a serem executadas pelos membros de uma equipe. Dessa forma, os princípios táticos e a qualidade da técnica irão ajudar a orientar a tomada de decisão dos componentes de uma equipe, que por sua vez será sucedida de uma ação de natureza técnicotática, buscando atender as necessidades da situação apresentada durante a partida ${ }^{12-13}$.

Estudos prévios em outras modalidades coletivas têm demonstrado que algumas açóes técnico-táticas são determinantes para o sucesso da equipe, além de serem dependentes da modalidade estudada. Por exemplo, enquanto Costa et al. ${ }^{14} \mathrm{e}$ Rocha e Barban$\mathrm{TI}^{15}$ apontam o jogo mais rápido (bola de primeiro

\section{Método}

\section{Participantes}

Foram analisadas duas temporadas de uma equipe de futsal de alto rendimento, participante da Liga Nacional da modalidade. A equipe analisada treinava em média seis dias por semana, com duas sessóes diárias e duração entre 80 a 120 minutos para cada sessão. Antes do início do estudo, os atletas foram informados do propósito da investigação e assinaram um termo de consentimento livre e esclarecido, aceitando participar da pesquisa, seguindo as normas de segurança resolução 196/96 do Conselho Nacional da Saúde. O estudo foi aprovado pelo Comitê de Ética de Pesquisa local sob o parecer no 266/2010. tempo) como um fator fundamental para o sucesso em ataques de voleibol, GABBET et al. ${ }^{8}$ indicam que a eficiência do passe e a habilidade para realizar desarmes diferenciam os jogadores de rúgbi profissionais de seus pares semi-profissionais. Por outro lado, no caso do futebol, RAmpinini et al. ${ }^{9}$ verificaram que as equipes com melhores colocaçôes na competição apresentavam melhores desempenhos, comparados àqueles com piores colocaçóes, em determinadas ações técnico-táticas específicas da modalidade realizadas por seus jogadores durante as partidas, como o maior número de passes curtos, passes completos, desarmes, dribles, chutes e chutes no alvo.

Levando em consideração os resultados apresentados por Rampinini et al. ${ }^{9}$, é plausível admitir que estas açóes possam interferir no resultado das partidas e, em consequência, poderiam ser utilizadas como indicadores de desempenho e até mesmo possíveis discriminadores do nível de qualificação de jogadores no esporte coletivo ${ }^{8}$. Entretanto, ainda náo está claro se as açôes técnico-táticas específicas da modalidade realizadas durante as partidas de futsal são capazes de discriminar, tanto o resultado de uma partida na modalidade futsal, quanto o nível destes jogadores.

Assim, considerando a importância de se investigar a frequência de ações técnico-táticas realizadas no futsal de alto rendimento, e buscando avançar o conhecimento dos fatores discriminantes de sucesso em partidas de futsal, o presente estudo teve como objetivo principal verificar se as açôes técnicotáticas específicas da modalidade seriam capazes de discriminar o resultado de partidas de futsal de alto rendimento para uma equipe determinada.

\section{Procedimentos}

Foram acompanhadas 51 partidas de uma equipe de futsal de alto rendimento ao longo de dois anos de disputa da Liga Nacional de futsal, das quais foram retidas para análise as açôes técnicas das 40 partidas em que a equipe apresentou o resultado de vitória ou derrota. Não foram consideradas as partidas terminadas em empate. Ao longo destas partidas, a equipe analisada obteve 18 vitórias (45\%) e 22 derrotas (55\%).

Um mesmo avaliador, com experiência de cinco anos em análise de jogos de futsal, foi responsável por realizar o "scout" técnico-tático de todas as 
partidas analisadas. O "scout" foi realizado "in loco" e durante o decorrer das partidas. Antes do inicio do estudo, 10 sessóes de treinamento com jogos de cinco contra cinco (quatro jogadores de linha mais um goleiro), simulando uma partida oficial, foram gravadas em uma câmera digital (Sony, Brasil) com frequência de $60 \mathrm{~Hz}$. O avaliador realizou o "scout" técnico "in loco" durante os treinamentos e posteriormente, a partir das imagens gravadas, um dia após a sessão de treinamento em questão. $\mathrm{O}$ coeficiente de variação esteve entre 0,5 e 4,2\%.

Durante cada partida, foram analisados os números totais das seguintes açóes técnico-táticas específicas da modalidade: Desarmes Realizados (DR), Desarmes Sofridos (DS), Total de Finalizaçóes Realizadas (TFR), Total de Finalizações Sofridas (TFS), Finalizaçóes Certas Realizadas (FCR), Finalizações Certas Sofridas (FCS), Passes Incompletos Realizados
(PIR) e Passes Incompletos do Adversário (PIA). As ações técnico-táticas selecionadas foram previamente estabelecidas pelos membros da comissão técnica da equipe analisada, os quais apresentavam um período de experiência de no mínimo cinco anos de disputa da Liga Nacional de futsal.

Além disso, foi realizada uma segunda análise buscando identificar se a execução de um maior número de determinada açáo técnico-tática em relação ao adversário seria capaz de discriminar o resultado de vitória e derrota. Para isso, foram calculados os índices de coeficiente para cada açâo técnico-tática, a partir da razão entre a ação realizada e a sofrida. Neste caso, valores acima de um representariam um maior número de açôes realizadas, enquanto valores abaixo de um representariam um maior número de açôes sofridas. O conceito de cada ação técnicotática está exposto no QUADRO 1.

QUADRO 1 -Conceito de cada ação técnico-tática específica da modalidade analisada durante as partidas de futsal.

\begin{tabular}{|l|l|}
\hline \multicolumn{1}{|c|}{ Variável } & \multicolumn{1}{c|}{ Conceito } \\
\hline Desarme & $\begin{array}{l}\text { Caracterizado por duas situações: } \\
\text { 1) atleta retira a bola do domínio do adversário, retomando ou não a posse de bola; } \\
\text { 2) atleta intercepta uma finalizaçáo do adversário. }\end{array}$ \\
\hline Total de finalizações & Número de finalizaçôes realizadas. \\
\hline Finalizaçóes certas & $\begin{array}{l}\text { Número de finalizaçóes realizadas que acertaram o alvo (1- gol; 2 - defesa do goleiro; 3 - } \\
\text { finalização na trave). }\end{array}$ \\
\hline Passes incompletos & Passes que não chegaram ao companheiro de equipe. \\
\hline Coeficiente & $\begin{array}{l}\text { Coeficiente = Ação realizada / Ação sofrida. } \\
\text { (Ex: Coeficiente de desarme = Desarmes Realizados / Desarmes Sofridos). }\end{array}$ \\
\hline
\end{tabular}

\section{Análise estatística}

Os dados foram apresentados com valores de média e desvio-padrão. $\mathrm{O}$ teste de KolmogorovSmirnov apontou que apenas a variável "coeficiente da finalização" apresentou uma distribuiçáo que se desviava da normal. Assim, para esta variável foi aplicada uma transformação logarítmica (Log), permitindo entáo contemplar o pressuposto da distribuição normal. O teste $\mathrm{M}$ de Box, empregado para avaliar a igualdade das matrizes de variância/ covariância das variáveis, indicou homoscedasticidade tanto para as variáveis absolutas quanto para os coeficientes. Com o intuito de se estudar a multicolinearidade, foram calculados os coeficientes de correlação de Pearson entre todas as combinações de variáveis para as duas análises. Como nenhuma situação de multicolinearidade perfeita foi encontrada e somente três correlaçôes se apresentaram moderadas, optou-se por não excluir nenhuma das variáveis da análise.

Para se atingir o modelo discriminante foi utilizado o método de estimaçáo simultânea, com a significância sendo verificada pelo teste de Wilk's Lambda. Foram considerados os valores de carga discriminante acima de 0,40 para seleção das variáveis de açóes técnicas no modelo. Também foi realizada uma análise de variância multivariada para amostras independentes, para comparação das médias de cada variável considerando o resultado da partida (vitória ou derrota). Todas as análises foram realizadas no "software" SPSS (v. 20.0, SPSS Inc., Chicago, IL, USA). O nível de significância foi estabelecido em $5 \%(\mathrm{p}<0,05)$. 


\section{Resultados}

Os resultados da análise mostraram que apenas as variáveis DR e DS apresentaram cargas discriminantes acima de 0,40 , indicando que as mesmas poderiam discriminar o resultado de vitória e derrota em partidas de futsal (TABELA 1). Foram observados valores de Wilk's Lambda de 0,39 , que indicaram a significância $(\mathrm{p}<0,05)$ e a capacidade discriminatória do modelo. Os resultados de validação cruzada foram utilizados para identificar observaçôes classificadas de forma equivocada. Assim, observou-se que este modelo foi capaz de classificar corretamente $77,5 \%$ dos casos analisados.

Apenas a variável Coeficiente de Desarme apresentou carga discriminante acima de 0,40 , quando considerados os índices de coeficiente para cada açâo técnico-tática, apontando que essa foi a única a discriminar o resultado de uma partida de futsal (TABELA 1). Foram observados valores de Wilk's Lambda de 0,47 , que indicaram a significância ( $p<$ $0,05)$ e a capacidade discriminatória deste modelo. Os resultados de validação cruzada apontam que este modelo foi capaz de classificar corretamente $77,5 \%$ dos casos analisados.

Os resultados da Análise multivariada de variância mostram diferenças para Desarmes Realizados, Desarmes Sofridos e Coeficiente de Desarmes entre os resultados de vitória e derrota (TABELA 2).

TABELA 1 - Carga discriminante das ações técnico-táticas específicas da modalidade realizadas durante as partidas de futsal.

DR:Desarmes Realizados; DS: Desarmes Sofridos; PIR: Passes Incompletos Realizados;

TFS: Total de Finalizações Sofridas;

PIA: Passes Incompletos do Adversário;

FCS: Finalizações Certas Sofridas;

FCR: Finalizações Certas Realizadas;

TFR: Total deFinalizações Realizadas.

* Cargas discriminantes acima de 0,40 .

\begin{tabular}{lccc}
\hline Açáo técnico-tática & Carga discriminante & Coeficiente das açóes técnico-táticas & Carga discriminante \\
\hline DR & $0,64^{*}$ & DR / DS & $0,94^{*}$ \\
DS & $-0,56^{*}$ & TFR / TFS & 0,18 \\
PIR & 0,22 & FCR / FCR & 0,11 \\
TFS & 0,21 & PIR / PIA & 0,08 \\
PIA & 0,18 & & \\
FCS & 0,11 & & \\
FCR & 0,11 & & \\
TFR & 0,05 & & \\
\hline
\end{tabular}

TABELA 2 - Comparação entre a frequência das ações técnico-táticas específicas da modalidade em situações de vitória e derrota durante as partida de futsal.

DR: Desarmes Realizados; DS: Desarmes Sofridos; PIR: Passes Incompletos Realizados;

TFS: Total de Finalizações Sofridas;

PIA: Passes Incompletos do Adversário;

FCS: Finalizações Certas Sofridas;

FCR: Finalizações Certas Realizadas;

TFR: Total de Finalizações Realizadas.

* Diferenças estatisticamente significantes da ação técnico-tática específica da modalidade comparada em situação de vitória ou derrota ( $p$ $<0,05$ ).

\begin{tabular}{lcc}
\hline \multicolumn{1}{c}{ Açáo técnico-tática } & Vitória & Derrota \\
\hline DR & $43,7 \pm 7,2$ & $31,5 \pm 8,2^{*}$ \\
DS & $29,2 \pm 7,7$ & $39,1 \pm 6,6^{*}$ \\
DR / DS & $1,57 \pm 0,39$ & $0,84 \pm 0,35^{*}$ \\
TFR & $27,0 \pm 5,7$ & $27,8 \pm 6,3$ \\
TFS & $24,2 \pm 7,6$ & $21,2 \pm 5,6$ \\
TFR / TFS & $1,22 \pm 0,48$ & $1,44 \pm 0,60$ \\
FCR & $16,6 \pm 5,0$ & $15,3 \pm 4,1$ \\
FCS & $14,2 \pm 5,5$ & $11,7 \pm 4,1$ \\
FCR / FCS & $1,35 \pm 0,70$ & $1,51 \pm 0,82$ \\
PIR & $44,3 \pm 7,7$ & $39,9 \pm 8,1$ \\
PIA & $41,5 \pm 8,6$ & $39,0 \pm 9,1$ \\
PIR / PIA & $1,10 \pm 0,27$ & $1,05 \pm 0,24$ \\
\hline
\end{tabular}




\section{Discussão}

O objetivo do presente estudo foi verificar se as açôes técnico-táticas específicas da modalidade seriam capazes de discriminar o resultado de partidas de futsal de alto rendimento. $\mathrm{O}$ principal resultado do estudo foi que os números absolutos das açôes técnico-táticas Desarmes Realizados (DR) e Desarmes Sofridos (DS) foram capazes de discriminar o resultado de uma partida de futsal. Além destes valores absolutos, constatou-se que a razão DR/DS também emergiu como um fator discriminante para o resultado da partida no futsal.

No que concerne aos valores absolutos das açóes técnico-táticas específicas da modalidade, pode-se observar que apenas DR e DS foram capazes de discriminar os resultados de vitória e derrota. Nota-se que estas variáveis de açóes técnico-táticas apresentaram altos valores de carga discriminante $(0,64$ para $\mathrm{DR}$ e - 0,56 para DS). Esses resultados indicam que além da relevância em se realizar um determinado número de desarmes ao longo da partida, atingir um maior número de DR comparado a DS também parece ser um fator fundamental para o resultado de vitória na equipe analisada, representado pela alta carga discriminante do Coeficiente de Desarmes $(0,94)$.

A consistência destes resultados é confirmada pela análise que apontou que as variáveis são capazes de classificar corretamente cerca de $80 \%$ dos casos de vitória e derrota investigados, indicando a pertinência e a importância destas variáveis em uma partida de futsal. Os maiores valores de DR apresentados na situação de vitória e maiores valores de DS na situação de derrota ratificam a importância destas açōes para se aumentar as chances de sucesso (resultado de vitória).

O presente estudo corrobora em parte com os achados de RAmpinini et al. ${ }^{9}$, analisando jogadores de futebol de campo das cinco melhores e cinco piores equipes classificadas no campeonato italiano. A investigação conduzida por RAMPININI et al. ${ }^{9}$ apontou que além do maior número de desarmes, um maior número de dribles, passes curtos, sucessos nos passes curtos, total de finalizaçóes e total de finalizações certas foram as ações técnico-táticas mais relevantes para o sucesso de equipes de futebol de alto nível ao longo de uma competição. Entretanto, vale ressaltar a divergência entre o resultado do presente estudo e o de Rampinini et al. ${ }^{9}$ no que diz respeito às açôes de finalização.

Essa divergência pode ser explicada, pelo menos em parte, por conta da presente investigação ter analisado apenas uma equipe e, dessa forma, o fato da ação de finalização nâo se apresentar como um fator discriminante do resultado da partida também pode estar associado à maneira de atuar da equipe investigada. Por outro lado, Rampinini et al. ${ }^{9}$ analisaram diferentes equipes, considerando assim uma maior variedade de interaçôes entre jogadores (intra: jogadores da mesma equipe; e inter: jogadores de equipes adversárias), o que por sua vez, também poderia acarretar em uma diferente frequência de ações técnico-táticas. Ainda nesse sentido, Hughes et al. ${ }^{16}$ por exemplo, mostraram que diferentes equipes de futebol do campeonato inglês apresentam características particulares para criação de oportunidades de gol.

Essa possível explicação reforça a importância de se considerar os restritores do ambiente (ex. nível e organização tática das equipes), na configuração de padróes emergentes de desempenho no esporte coletivo ${ }^{17}$. Indica também a necessidade de se avançar o conhecimento do efeito de se restringir taticamente as dimensôes do jogo (ex. defendendo com maior ou menor pressão, atacando com maior ou menor distância entre os jogadores do mesmo time e dos seus oponentes) na frequência e eficiência das açôes técnico-táticas, oportunidades de finalizaçáo e gols marcados, e o subsequente sucesso ou insucesso em partidas de futsal, a partir da compreensão da interaçấo dinâmica dos elementos do jogo ${ }^{18}$.

Interessantemente, a capacidade de realizaçáo de desarmes também foi destacada em um estudo com rúgbi e possibilitou discriminar jogadores titulares, reservas e não selecionados para a Liga profissional. Os resultados da investigação de GABBET et al. ${ }^{8}$ indicam que a eficiência do passe e a habilidade para realizar desarmes diferenciam os jogadores de rúgbi profissionais e semi-profissionais. Esses resultados em conjunto salientam, portanto, a importância das açôes de desarme para as modalidades coletivas, notadamente no que se refere ao futebol, ao rúgbi e, agora, para esta equipe analisada no futsal.

É pertinente se admitir "a priori” que o número de desarmes pode ser considerado um indicador de eficiência do sistema defensivo da equipe. Os resultados do presente estudo sustentam esta suposiçáo e indicam a importância de adoçáo de um sistema defensivo que consiga alcançar um determinado nível de eficiência durante a partida. Em especial, que seja mais eficaz que o sistema ofensivo da equipe adversária. Essa poderia ser uma das formas de evitar a quebra da estabilidade do sistema - interação ataque e defesa - e dificultar as penetraçôes dos atacantes e, em consequência, reduzir as chances de finalização em claras condições de marcar o gol. 
Deve-se, portanto, ressaltar a importância de um adequado desenvolvimento do sistema defensivo em equipes de futsal de alto rendimento, tanto no que se refere à organização tática como no tocante à técnica adequada para realização do desarme, com intuito de se aumentar as possibilidades de sucesso competitivo das mesmas. Este apontamento, por sua vez, levanta a necessidade de mais investigaçôes sobre a temática relacionada às formas de treinamento que se apresentem mais eficientes para o aperfeiçoamento do sistema defensivo.

No presente estudo, as ações técnico-táticas de passe e finalização não se mostraram capazes de discriminar o resultado de vitória e derrota em partidas de futsal da equipe analisada. Como exposto anteriormente, esses resultados divergem de investigaçóes anteriores no rúgbi ${ }^{8}$ e no futebol ${ }^{9}$. Mais uma vez, a maneira de atuar da equipe analisada pode ser um fator plausível para explicar essa diferença.

A ausência de uma abordagem que contemple a interação entre as equipes participantes na análise dos resultados do presente estudo, apesar de não poder ser considerada como uma ameaça aos resultados aqui observados, pode ser vista como uma limitação na perspectiva ecológica da análise de jogo no esporte coletivo $^{18-19}$, pois não levou em conta a inter-relação de cooperação e oposiçãa. Outra limitação do presente estudo é que somente uma equipe foi analisada no tocante as açôes técnico-táticas específicas da modalidade. Apesar desta limitação, o fato de se investigar duas temporadas consecutivas desta equipe no principal campeonato da modalidade no Brasil, durante 40 partidas, é um aspecto relevante e um dos pontos fortes da presente investigaçáo. Portanto, é razoável admitir-se que os resultados obtidos no presente estudo e as inferências sobre os fatores técnicotáticos discriminantes do sucesso em partidas de futsal podem ser referentes a um estilo próprio de jogo adotado pela equipe investigada e náo necessariamente seria reproduzido em outras equipes. Deste modo, é recomendável que novas investigaçōes com abordagem semelhantes sejam realizadas em outras equipes de alto rendimento, durante a Liga Nacional, para que os resultados possam confrontados. Estudos em Ligas Internacionais, com delineamentos similares, poderiam também possibilitar a comparação entre estilos de jogo e fatores discriminantes de sucesso e de desempenho entre diferentes países.

Os resultados do presente estudo permitem concluir que a eficiência em relação ao número de Desarmes Realizados e de Desarmes Sofridos pode ser um fator importante para resultado de vitória em partidas de futsal de alto rendimento. Assim, este estudo aponta que as açóes técnico-táticas de desarme referentes ao sistema defensivo de determinada equipe de futsal podem discriminar o resultado da partida, bem como para a importância do sistema ofensivo no que diz respeito a uma melhor organização do ataque e, consequentemente, uma menor possibilidade de sofrer desarmes.

\section{Abstract}

Wich technical-tactical actions performed in futsal matches can discriminate the result of winning or defeat?

The objectives of this study were to verify whether specific technical-tactical actions of futsal would be able to discriminate the result in high-performance futsal matches. Forty matches from one futsal team were analyzed over two National League seasons, in which the assessed team showed the result of winning or defeat. For each match, the number of total technical-tactical actions were analyzed: Tackles done, Tackles suffered, Total shots done, Total shots suffered, Shots on target done, Shots on target suffered, Incomplete pass done, Opponent incomplete pass; as well coefficient indexes for each technical-tactical action, from the ratio between action done and suffered. Discriminant Analysis was realized using simultaneous estimation method. Only Tackles done, Tackles suffered and Tackles coefficient showed discriminating loads above 0.40 , which indicate that these variables can discriminate the result of winning and defeat in futsal matches. It can be concluded that efficiency related to Tackles done and Tackles suffered is a determinant point to achieve the winning in high-performance futsal match. This study shows that the defensive system in a futsal team, in which must be considered both tactical organization and appropriate technique to perform the tackle could discriminate the match's result in the analyzed team. Offensive system is also important in order to provide a better attack organization, and consequently, a lower possibility to suffer a tackle.

KEY WORDS: Performance analysis; Team sports; High-performance sports; Sports performance; Futsal. 


\section{Referências}

1. Castagna C, D’Ottavio S, Vera JG, Barbero-Alvarez JC. Match demands of professional futsal: a case study. J Sci Med Sport. 2009; 12:490-4.

2. FIFA. Fédération Internationale de Football Association. Suporte técnico. [citado 11 dez. 2012]. Disponível em: www.fifa.com.

3. Barbero-Alvarez JC, Soto VM, Barbero-Alvarez V, Granda-Vera J. Match analysis and heart rate of futsal players during competition. J Sports Sci. 2008;26:63-73.

4. Makaje N, Ruangthai R, Arkarapanthu A, Yoopat P. Physiological demands and activity profiles during futsal match play according to competitive level. J Sports Med Phys Fitness. 2012;52:366-74.

5. Dogramaci SN, Watsford ML, Murphy AJ. Time-motion analysis of international and national level futsal. J Strength Cond Res. 2011;25:646-51.

6. Pedro RE, Milanez VF, Boullosa DA, Nakamura, FY. Running speeds at ventilatory threshold and maximal oxygen consumption discriminate futsal competitive level. J Strength Cond Res. 2013;27:514-8.

7. Barbero-Alvarez JC, D’Ottavio S, Vera JG, Castagna C. Aerobica fitness in futsal players of different competitive level. J Strength Cond Res. 2009;23:2163-6.

8. Gabbett TJ, Jenkins DG, Abernethy B. Relative importance of physiological, anthropometric, and skill qualities to team selection in professional rugby league. J Sports Sci. 2011;29:1453-61.

9. Rampinini E, Impellizzeri FM, Castagna C, Coutts AJ, Wisløff U. Technical performance during soccer matches of the Italian Serie A league : Effect of fatigue and competitive level. J Sci Med Sport. 2009;12:227-33.

10. Iaia FM, Rampinini E, Bangsbo J. High-intensity training in football. Int J Sports Physiol Perform. 2009;4:291-306.

11. Ramos V, Tavares FJS. A seleção dos jovens atletas de basquetebol: estudo com técnicos brasileiros. Rev Bras Cineantropom Desempenho Humano. 2000;2:42-9.

12. Greco PJ, Benda R. Iniciação esportiva universal. Belo Horizonte: UFMG; 1998.

13. Costa IT, Silva JMG, Greco PJ, Mesquita I. Princípios táticos do jogo de futebol: conceitos e aplicação. Motriz. 2009;15:657-68.

14. Costa G, Mesquita I, Greco PJ, Ferreira NN, Moraes JC. Relação entre o tempo, o tipo e o efeito do ataque no voleibol masculino juvenil de alto nível competitivo. Rev Bras Cineantropom Desempenho Hum. 2010;12:428-34.

15. Rocha, MA, Barbanti VJ. Análise das açôes de saltos de ataque, bloqueio e levantamento no voleibol feminino. Rev Bras Cineantropom Desempenho Hum. 2007;9:284-90.

16. Hughes M, Dawkins N, David R, Mills J. The perturbation effect and goal opportunities in soccer. J Sports Sci. 1998;16:20.

17. Vilar L, Araújo D, Davids K, Button C. The role of ecological dynamics in analysing performance in team sports. Sports Med. 2012;42:1-10.

18. Gréhaigne J-F, Bouthier D, David B. Dynamic-system analysis of opponent relationships in collective actions in soccer. J Sports Sci. 1997;15:137-49.

19. McGarry T, Anderson DI, Wallace SA, Hughes MD, Franks MI. Sport competition as a dynamical self-organization system. J Sports Sci. 2002;20:771-81.

\section{Agradecimentos}

Os autores gostariam de agradecer ao Petrópolis Esporte Clube pela anuência para a condução da investigação realizada.

ENDEREÇO

Bernardo Miloski

R. Prof. Stoeller, 1176

25680-502 - Petrópolis - RJ - BRASIL

Recebido para publicação: 07/08/2013

Revisado: 11/12/2013

e-mail: bernardomiloski@yahoo.com.br

Aceito: 20/03/2014

Rev Bras Educ Fís Esporte, (São Paulo) 2014 Abr-Jun; 28(2):203-09 • 209 